\title{
THE ISOLATION OF AN ACTINOMYCETES-LIKE ORGANISM FROM ROOT CANALS ${ }^{1}$
}

\author{
MARY C. CROWLEY, MSPH
}

School of Dentistry, University of Michigan, Ann Arbor, Mich.

The actinomycetes are a group of fungi, usually classified as hypomycetes, probably bearing a relationship to the acid fast and diphtheroid groups of bacteria. The organisms are commonly found in the soil, and certain types may produce lesions in animals and man. In man the type most often isolated from lesions is Actinomycetes bovis with primary lesions about the head and neck in approximately half the cases studied. The infection in man is commonly called "lumpy jaw," characterized by a purulent discharge containing "sulfur granules" and the formation of sinuses and granulation tissue. At the periphery of the lobulated granules are radiating clubs, composed of mycelia slightly thicker at the tip, surrounded by sheaths which take the acid rather than basic dyes. The name "ray fungus" has been given to the actinomycetes because of the appearance of the granules, when examined under the microscope. The club forms are usually seen in tissues, although a few workers have reported formation of clubs in artificial media $(1,2)$. The granules when crushed and stained are seen to be made up of extremely fine, branching gram positive filaments. When first isolated, the organisms grow best under anaerobic or partially anaerobic conditions, but upon further cultivation most strains will grow aerobically on suitable media.

The mode of infection of the disease is not exactly clear. It is more common among farmers and those who handle grains, straw, etc. For this reason it is thought that the organisms are carried on grains and other vegetable matter to the mouth. The infection in cattle and in man is believed to be caused by the same organism.

Wright (3) suggested that Actinomycetes bovis was a normal inhabitant of the secretions of the buccal cavity and intestinal tract, both

\footnotetext{
${ }^{1}$ Read at the 17th General Meeting of International Association for Dental Research, Cleveland, Ohio, March, 18 and 19, 1939 (J. D. Res., 18: 267, 1939). (Received for publication, February 16, 1941.)
} 
of man and animals. He prophesied that it would be found in fragmented filaments with other bacteria and not differentiated from them. He further states that the part played by foreign bodies is not that of a carrier of the organisms, but that they serve to traumatize the tissues so that the organisms already present develop into characteristic granules and produce the lesions which we call actinomycosis.

Lord (4) studied the contents of carious teeth and tonsils of patients having no indications of mycotic infection. Serial sections made of the material from some of the cases showed what the author considered to be typical actinomycetes. Animals inoculated intraperitoneally, developed omental tumors with formation of club-like structures resembling the ray fungus. Smear preparations from dental cavities showed gram staining filaments, slender and wavy, both long and short forms with branching in only rare instances. Lord did not report the isolation of the organism in pure culture.

Davis (5) described small granules with ray-like structures, resembling in some respects granules of actinomycetes that he isolated from tonsillar crypts. He stated that these granules have been repeatedly mistaken for actinomycetes, but in reality they are very different. $\mathrm{He}$ found that these granules were made up of filaments along which were arranged fusiform bacilli spirochetes and cocci in a typical test tube brush arrangement. He reported a similarity between these lesions and those described by Lord. However Davis did not see the clubs definitely described by Lord. Davis further stated that experimentally, intraperitoneal injections of tonsillar granules produced lesions with structures simulating true actinomycetes. Henrici (6), stated that the actinomycetes reported as present in tonsils, pyorrhea pockets and apical abscesses are probably the granules reported by Davis. Tunnicliff and Jackson (7) have succeeded in cultivating these forms anaerobically, the cultures showing the typical test tube brush arrangement. They have named the species Vibriothrix tonsillaris.

Emmons $(8,9)$ reported the cultivation of a saprophytic actinomycetes from teeth and tonsils. He classified the organisms as Actinomyces bovis. Lord and Trevett (10) reported the isolation of 4 strains of actinomyces from surfaces and contents of carious teeth and stated that they were like Actinomyces bovis.

In the dental clinic at the University of Michigan direct smears 
of material removed from root canals have occasionally shown short, branching, gram positive filaments which did not grow in cultures. Recently strains of an organism similar to that seen in direct smears have been isolated from root canals of (7) clinic patients with following case histories. All of these patients were negative as regards systemic infection.

Case I. (C. C.) A 14 year old boy from a rural community had had a blow on the face in the incisor region 2 years before he came to the clinic. Five teeth had devitalized pulps with radiographic evidence of periapical tissue destruction. Only one tooth yielded actinomycetes-like organisms.

Case II. (B. G.) A student about 21 years of age gave a history of receiving a blow in the incisor region about 5 years previously. One of the incisors was discolored and showed a slight swelling over the apical region. Radiographs demonstrated an apical area of translucency.

Case III. (S. L.) Patient was also a student, age 19 years, who came to the clinic because of a toothache due to vital exposure of the pulp.

Case IV. (E. G.) Patient was a student about 22 years of age. He had received a blow across the mouth in an automobile accident 7 years previously. The pulp of the tooth studied was also exposed to caries. Another tooth had been treated 5 years after the accident because of loss of vitality. At that time direct smears had shown gram positive branching filaments which did not grow in cultures.

Case V. (A. G.) Patient was an instructor in the German Department in the University. His history was that of recurrent caries under an old amalgam filling. Radiographs showed a large area of tissue destruction.

Case VI. (P. L.) Patient was an employee in the University Shops who came into the clinic because of a pulp exposure. X-rays showed a slight thickening of peridontal membrane at the apex of the tooth.

Case VII. (F. E.) Patient was a man about 53 years of age who gave a history of pulp death due to a large crown approximating the pulp.

In none of these cases was the organism recovered in pure culture. Three of the patients with histories of trauma had received the injury some time ago, 1 of these and the remaining 4 had open carious lesions allowing the entrance of mouth secretions. The apical lesions in no case resembled a mycosis so that the organisms if related to the actinomycetes were present as saphrophytes. Case IV showed the organisms in direct smears 2 years before they were isolated yet in this time no mycotic lesions had appeared.

\section{METHOD OF ISOLATION}

The 7 strains all presented very similar cultural characteristics so that they will not be considered separately. Cultures were all isolated from root canals in a beef infusion broth $\mathrm{pH} 7.4$ containing 
0.1 per cent agar, 0.2 per cent glucose and 5 per cent ascitic fluid. This media allows the growth of some anaerobic as well as aerobic organisms. All strains were grown at $37.5^{\circ} \mathrm{C}$. Growth appeared on the second day as a faint diffuse cloud around the paper point used to inoculate the media. Quite often it was necessary to incubate cultures a day or 2 longer to be certain of growth. When material from the semi-solid media was transferred to a blood agar plate, growth sometimes appeared in 24 hours, but more often 48 hours, as very minute dew-like colonies.

After the first isolation, all the strains grew well on blood agar, glucose beef infusion agar and plain beef infusion agar, beef extract agar containing starch, as well as beef infusion bouillon, $\mathrm{pH} 7.4$. Strains cultured aerobically at $37.5^{\circ} \mathrm{C}$. grew well in 2 days' time. Czepak's medium, a synthetic medium used for the cultivation of actinomycetes, did not give consistent results although sucrose, dextrose, and starch were used as the source of carbohydrate and plates were incubated anaerobically as well as aerobically. Those colonies which did develop were so extremely minute as to be barely visible to the naked eye. They were always few in number and transfers made of these colonies to the same medium, and blood agar, failed to grow.

Colonies on all bacteriological media used were discrete and creamy white in color and after 3 or 4 days' incubation usually showed wrinkled surfaces, some colonies appearing as rosettes. The colonies were extremely tenacious and seemed to send root-like projections into the media. Some colonies were "rougher" than others, and when examined under the microscope branching filaments were seen coming out from the edge of the colony. The colonies were similar to a stock strain of Actinomycetes bovis. In broth the organisms grew in 3 days as flakes in the bottom of the tube with no clouding of the media. After 3 to 4 days incubation, growth became rather viscid in some strains. Growth at room temperature $\left(23^{\circ}-24^{\circ} \mathrm{C}\right.$.) on blood agar plates occurred in 3 to 4 days which was slower than that at $37.5^{\circ} \mathrm{C}$.

Strains when tested on 3 per cent starch beef extract agar grew extremely well but gave no evidence of diastatic action when tested with iodine solution. When grown in $\mathrm{pH} 5.7$ beef infusion broth 
1 per cent glucose after 6 days' incubation, no change in $\mathrm{pH}$ was noticed, but growth was scant; grown in $\mathrm{pH} 6.8$ beef infusion broth, no glucose added, did not vary the $\mathrm{pH}$. No pigment was produced nor were aerial mycelia seen on any medium. When grown in beef infusion broth containing 10 per cent gelatin, growth occurred with only 3 strains after 8 days' incubation. The 3 strains did not liquefy gelatin.

Smears made of both colonies and broth cultures showed non-acid fast, gram positive, fine, branching filaments. The filaments measured from $.5-.75$ microns. Tips of filaments were very often enlarged to give a club-like appearance but this is not the typical club seen in granules of actinomycetes from lesions. The filaments were much shorter than those of the stock strain of Actinomycetes bovis but the actinomycetes-like organism had been rapidly subcultured, a condition which Henrici (11) states causes the fragmentation of the mycelium, resulting in bacterial-like growth. Small enlargements at the tips of filaments or rods, which Wright (12) describes in his cultures of actinomycetes as "pear or olive-shaped swellings" could be seen in most of the strains. Coccal forms were seen in some of the older cultures.

\section{ANIMAL INOCULATION}

Four animals which were inoculated with 4 strains, intraperitoneally, were killed after 18 days and showed no pathologic lesions on autopsy and sectioning.

\section{CONCLUSIONS}

The organisms isolated bear a similarity to true actinomycetes and seem to fulfill Wright's prophesy that the organisms would be found in the oral cavity as fragmented filaments. The organisms are similar to those reported by Emmons and Lord, whose observations are here confirmed. (If these organisms are true actinomycetes, they would seem to fill a rôle analogous to the organisms of Vincent's infection which are present in the mouth without necessarily causing infection.)

The organisms were similar to Actinomycetes bovis, in that they were isolated with some difficulty, growing under partial anaerobic conditions and only in media containing body fluids. They formed 
branched mycelia with structures similar to the true bovis. After primary isolation, they grew more rapidly than most strains of actinomycetes of human origin, and did not hydrolize starch. (Growth on Czepak's media was doubtful which is not true of the Actinomycetes bovis.)

The organisms are interesting because they seem to be similar to Actinomycetes bovis and yet are not responsible for any lesions.

\section{REFERENCES TO LITERATURE}

1. Bayne-Jones, S., J. Bact., 10: 569, 1925.

2. GrBson, Joan, J. Path. \& Bact., 39: 533, 1934.

3. Wright, J. A., J. Med. Research, 13: 4, 1905.

4. Lorn, F. T., Boston Med. \& Surg. J., 163: 82, 1910.

5. Davis, D. J., J. Inf. Dis., 1: 144, 1914.

6. Henrici, A. T., Molds, Yeasts and Actinomycetes. (New York, John Wiley: 1930) p. 261.

7. TunNiclifr, R. AND Jackson, J. Inf. Dis., 64: 12, 1930.

8. Emmons, C. W., Puerto Rico J. Pub. Health \& Trop. Med., 11: 63, 1935.

9. Idem, ibid, 11: 720, 1936.

10. LoRd, F. T. AND Trevetr, J. Inf. Dis., 58: 115, 1936.

11. HeNrIC, Molds, Yeasts and Actinomycetes. (New York, John Wiley: 1930)p. 240, fig. 42.

12. Wrigrt, J. A., J. Med. Research, 13: 4 (fig. 47), 1905. 